cillin and sulphonamide is that the effects of treatment are much more beneficial in the younger age groups. In elderly patients the pneumonia is often superposed on some underlying chronic disease, and in this type of case the resistance is at a low $\mathrm{ebb}$, so that the powers of recuperation may not prove sufficient to enable the patient to withstand the infection. Care must therefore be exercised in framing a prognosis, and the history of the previous health in the immediate past should be taken into account.

Of recent years it has been recognised that the commonest acute inflammatory consolidation of the lung is not true pneumonia, but pneumonitis ("primary atypical pneumonia"). The clinical distinction is easy to make because the respiration rate in pneumonitis is little raised. It is very important that the correct diagnosis should be made and that a loose diagnosis of "pneumonia" should be avoided, for the prognosis in pneumonitis is excellent and very little treatment is needed. It is not yet certain whether the condition is always due to a virus infection or whether it may on occasion result from a pneumococcal infection of exceptionally low virulence, but it is possible that both types of cases may be at present included in the one group. The treatment is always general and is on the lines already laid down in the first part of this article. It is generally agreed that the sulphonamide group of drugs is not effective in pneumonitis and, in any case, the illness tends towards spontaneous recovery with few complications. Of the drugs recommended perhaps the only one which is likely to be beneficial is quinine, and this is always worth a trial. The action of penicillin in pneumonitis is as yet unknown, but it would hardly seem likely that so potent a drug will ever be really needed in a condition which runs so benign a course.

\section{THE DIAGNOSIS AND TREATMENT OF PNEUMONIA IN INFANCY AND CHILDHOOD}

\section{By W. G. Wyllie, M.D., F.R.C.P.} (Phys. Hospital Sick Children, Great Ormond Street)

First let it be clearly understood that pneumonia in early childhood is often a preventable disease. It is among the children of the poorer classes that the bulk of the case-incidence occurs. In any local hospital one comes to know too well the districts, the families, from which one or more sibs, infants or toddlers, are admitted for treatment, some with recurrent attacks. Social medi- cine, or the study of positive health, will have great part to play in aiming at better housing proper clothing and feeding, dispelling ignoranc్ㅛㅇ on food values, and providing an adequate vitamire intake. The natural resistance of the ill-fed under-nourished, under-vitaminised child is below? par, and though florid rickets is now seldom seenc its old associate the chronic catarrhal state of the upper respiratory passages is still too common By loading bacterial infection on lowered resist $\mathbb{\square}$ ance, the catarrhal state is readily transformed into a pneumonia.

A further word on prevention: it may be denied that the prenatal diet of the mother affects the health of the infant unless extreme deficiencies are present. Yet Ebbs, Tisdall and Scott have given strong reasons to believe that by providing. expectant mothers, having a low income and on poor diet, with dietary supplements, the incidences of illness in their infants up to six months of age्f can be reduced. In a control group without supple ments to the maternal diet infection was present in $3 \mathrm{I}$ per cent of the infants as against 8 per cent oP infants of mothers on supplemented diets. In the control group the types of illness in the infants up to six months of age in order of frequency werद्ध anaemia, frequent colds (catarrh), dystrophy? rickets, pneumonia, and bronchitis. The figste three or four of these prepare the ground for easy inoculation of the bacterial infections.

When it is stated that pneumonia has its highese mortality at the two extremes of life, the states ment as it affects infancy must be qualified. It is the debilitated infant or young child whose chances of recovery are reduced, as its pneumonia is $\mathbb{2}$ complication of ill-health and often of measles o $\overrightarrow{\vec{b}}$ of whooping cough. These are the cases with $\vec{\not}$ high mortality in which chemotherapy often dis? appoints. But with an uncomplicated pneumonia a previously healthy infant in my experience has as good a chance of recovery by chemotherapy as the older child or adult.

The traditional division of pneumonia int lobar and broncho-pneumonia as separate entitie is losing ground, and is even passing from the latest text-books-a sure sign of decay! It is better to speak of Primary and Secondary pneu $\rightarrow$ monia, in either of which the anatomical distri․ㅡㄹ bution may take the lobar or the bronchial formin their incidence varying at different ages. Broncho= pneumonia is especially a disease of early infancys even as a primary. pneumonia; afterwards mores and more primary cases take the lobar formo The primary pneumonia is caused by the inhalation of recoverable or unknown bacteria and viruses币 Most often the noxus is a member of the pneumo? cocci, less often a haemolytic streptococcus or ao haemophilus influenzae, rarely the staphylococcus 
aureus. It is doubtful whether virus pneumonia in infants and children has been clinically detected in this country.

In a group of 95 pneumonias in infants (57) and young children (38) described by Ormiston et al., there were 66 primary cases, and of these 4I were primary bronchial and 25 primary lobar. The remainder were mostly bronchopneumonias secondary to measles and pertussis. The high figure of $4 \mathrm{I}$ primary bronchial pneumonias is explained by the fact of 32 being in infants below two years and 9 only in older children, but all below the age of five years. Under one year all the infants with one exception had anatomically the bronchial type of distribution. All cases at all ages complicating pertussis were bronchial pneumonias, while the association with measles in 20 cases were I6 bronchial and 4 lobar.

\section{Anatomical peculiarities of the infant and young child}

The calibre of the trachea, large and small bronchi, is very small in infants and young children. The bronchi of all sizes are more easily obstructed by plugs of mucus, and it follows that lobar and lobular collapse is a much more common event. By frequent radiography of children's chests in hospital it is not uncommon to discover accidentally a collapsed lobe or patch of collapse and consolidation in an apparently healthy child. Perhaps the collapse has disappeared by the time of the next X-ray in a few days or weeks. Such collapses occur more often than is suspected following tonsillectomy. These are as likely to be a result of the anaesthetic as of the operation. The uninfected mucus plug is a common cause of this occurrence.

Following a pneumonia in children the affected pulmonary area is apt to become a locus minoris resistentiae. One often finds in subsequent attacks of influenza, infectious fevers, pneumonia, or even by follow-up X-rays without the health of the child being obviously altered, that the area of the original insult is again in a state of collapse and consolidated, or that a whole lobe primarily affected is collapsed.

The relative softness of the chest wall in the infant permits of much greater recession of the ribs when the balance between external and internal pressure is upset by the presence of large areas of inactive pulmonary collapse. So in the debilitated under-nourished infant lacking vitamins attacked by bronchopneumonia, gross deformity of the chest may develop rapidly with sternal protrusion and costal recession bordering the sternum on either side and horizontally along the costal margins.

\section{ICLINICAL TYPES AND SYMPTOMS}

In primary pneumonia whether of lobar or lobular distribution, the onset is sudden. The younger the child, the less likely is cough to be present and sputum is rarely expelled, usually being swallowed. If sputum collects it is more often vomited, which is an early and common symptom. Fever rises quickly to 102 or $105^{\circ} \mathrm{F}$., the respiratory rate reaches 60 to 90 per minute, and the pulse rate accelerates to I4O or over. There is a malar flush on one or both cheeks, the alae nasi move with inspiration, and the respiration seems to be reversed to expiration-inspiration. The child feels hot and looks toxic. With an upper lobe involvement nuchal rigidity or head retraction may be present, and with lower lobe involvement abdominal pain may be complained of due to diaphragmatic irritation.

In the acute stage physical signs, dullness, tubular breath sounds, crepitations, may be obvious or lacking. Obvious, when the alveolar spaces either of lobar or lobular distribution are affected; lacking, when the inflammatory process remains a bronchiolitis and successive X-rays fail to reveal any parenchymatous shadows. Least common is the more proximal type of acute tracheitis, most alarming because of the inspiratory stridor and obstructed inspiration, with marked inspiratory recession of the costal margins. Frome this latter type pharyngeal obstruction by acute tonsillitis, diphtheria, foreign body must be excluded. In the ordinary form of acute primary pneumonia the diagnosis on symptoms alone is commonly simple. Bronchial asthma, however, of sudden onset, though usually self-terminating in one or two days, may simulate acute pneumonia, and it may be alleged that a child has had "ten or so attacks of pneumonia in a year."

It is in the secondary class that most of the hazardous and obstinate bronchopneumonias occur. They complicate whooping cough and measles, states of debility, gastro-enteritis in the infant, and themselves are liable to be complicated by otitis media and empyema. Physical signs are profuse, the areas of lobular collapse and consolidation shift from day to day, hour to hour, secretion is plentiful, and the chief risk occurs when insufficient unaffected lung tissue remains for the oxygenation of the blood. Either suffocation from anoxaemia, or drowning in its own secretions, are the two perils encountered in a severe case. There are other less common cases with an almost normal temperature chart, but with respirations up to Ioo or over and accelerated pulse rates, where percussion and auscultation detect shifting areas of dullness and rhonci, and X-rays patchy flocculent shadows. The appearance of the child may not suggest a serious illness, but anaemia and debility 
are certainly present. The course of the illness may be prolonged for weeks in all these obstinate wandering cases of bronchopneumonia migrans.

\section{TREATMENT OF THE ACUTE PNEUMONIAS}

Chemotherapy has greatly reduced the deathrate of all ages. For example, J. A. V. Davies, of Harvard, employing a sulphonamide, had only 5 deaths among $7 \mathrm{I}$ infants, and none of the failures could be ascribed solely to failure of chemotherapy, and I death, a case complicated by a massive empyema, among 79 older children. One is not allowed to see the eagerly awaited thermal crisis of former days of the lobar type of pneumonia. With sulphonamides the temperature can be expected to fall to normal within twenty-four to forty-eight hours usually, the respiration and pulse rate following soon afterwards. Physical signs and X-ray shadows more gradually disappear. The complication of empyema is much less often seen, though small collections of fluid often culturally sterile - the organism having been scotched by the sulphonamide-are not uncommonly discovered either by percussion or radiography. Even a massive pleural effusion is occasionally seen from which after repeated cultures a streptococcus or pneumococcus may be isolated. Such cases are liable to be mistaken for tuberculous pleural effusions, but a negative Mantoux test helps to distinguish them. In rare instances, even in earliest infancy, the staphylcoccus sureus may be the invading organism. Empyema is likely to occur and blood culture may be positive. Recoveries under massive dosage of sulphathiazole have been obtained. For the general run of cases of primary pneumonia sulphamezathine is a suitable drug, as it seldom causes vomiting or other minor toxic effects. In infants a suitable dosage is $3 \mathrm{gm}$. daily for three days, doubling the initial dose from $\frac{1}{2}$ to I gm. and $2 \mathrm{gm}$. for the two following days; for children over five years of age larger doses are required.

In the secondary pneumonias, complications of some other disease, the sulphonamides are less satisfactory. The antibody response of the patient is poorer. One or more courses of sulphonamide may be given, keeping the white blood-cell count and a liberal fluid intake under observation, but in these cases greater help in saving life is often obtained by the use of an oxygen tent. Typing of pneumococci and the use of specific sera are seldom employed in this country.

Cough mixtures are rarely required except by the chronic bronchopneumonia, when, if sputum judged by the crepitations is plentiful, or coughed up with difficulty, a mixture containing Tr. Ipecac. m.v., Amm. Carbonatis gr. $\frac{1}{4}$, and Spt. Ammon. Aromat. m. iii may aid in expelling it.
For the infant dangerously ill, dyspnóeic, in the气̊ oxygen tent, with signs of excessive intrabronchial 3 and alveolar exudate it is wiser to attempt toळ reduce the flow, and a four-hourly hypodermic of:. Strychnine gr. I/200-I/I50, Atropine gr. I/200$\mathrm{I} / \mathrm{I} 5 \mathrm{O}$, Adrenalin $\mathrm{m}$. I to 3 , alternating with ro? to 20 drops of brandy by mouth, often gives relief.

\section{THE CHRONIC PNEUMONIAS, BRONCHIECTASIS}

There are among the child population to-dayos many with unrecognised pulmonary crippling, the outcome of previous pneumonias, measles or whooping cough, who are casually labelled as $\vec{\omega}$ case of "bronchitis." This is a dangerous title as it only describes a symptom, is rarely a primaryo condition, but usually an accompaniment or complication of upper respiratory or tonsillar $\stackrel{-}{-}$ infection, rickets, bronchial asthma, or of a chronicin pulmonary lesion. Our conception of many pul-o monary diseases, bronchiectasis, pulmonary col- $\vec{\nabla}$ lapse, has been revolutionised within the lasto twenty years or so by the extending use of radiography, bronchoscopy, and bronchography withe an opaque substance-lipiodol. Now we recognise that an apparently satisfactory immediate recoveryo judged by absence of symptoms following $a^{2}$ pulmonary incident is no guarantee that collap $8, \overrightarrow{0}$ fibrosis, bronchiectasis, is not in existence. Theor younger in years, the more vulnerable are to. lungs to damage, often acquiring permanent scars liable to progressive deterioration at any age by subsequent re-infection or unsuitable employment.\$ In cases of chronic lung disease in adults, enquiry这 often elicits the fact of a sequence of pulmonary traumas dating from childhood.

The presenting symptom in all these chronic cases is cough, a loose productive cough. In children sputum is swallowed, not coughed out except when the quantity is excessive. Clubbing of the fingers is more a sign of the degree of purulent: infection in dilated bronchi than of chronicity of 3 . the pulmonary lesion.

The loose cough plus the history of earlier pulmonary infection, alone or complicating measles or pertussis, directs one to X-ray the child's chest, when one usually finds opacities, such as collapse of a lobe usually left or right lower, right middle, left lingula, or widespread radiatingo streaks, suggestive of possible bronchiectasis.

With such signs present, a thorough investigation is indicated at a chest-unit. When from $\omega$ the history a collapse is less than a year old, ito may be possible by bronchoscopy with suctione to remove excess mucus, which acting as a pluges in the narrowed lumen of an inflamed bronchus aids in maintaining a state of collapse in the dista alveoli. Next a bronchogram enables one to 
ascertain 'whether bronchial dilatation saccular or fusiform has occurred and its degree of involvement in the different lobes. Not all cases of bronchial dilatation, if treated early, are irreversible. The necessity for all cases is a prolonged period of fresh-air treatment. Later reassessments by X-ray and bronchogram will decide whether in the persistent case lobectomy offers the best chance of improvement.

Lobectomy nowadays offers good or improved health to many of these pulmonary cripples among children, and has a rapidly diminishing mortality. It is wise to look, however, to the beginnings of these pulmonary insults, and in any child after either a pneumonia, or measles, or whooping cough with pulmonary complications, to X-ray the child's chest. Only so can we hope to be early on the track of pulmonary damage, much of which is preventable.

(In this article any reference to tuberculous conditions of the chest in children is purposely omitted.)

\section{REFERENCES}

EBBS, J. H., TISDALL, F. F., and SCOTT, W. A. (I94I), Journ. of Nutrition, 22, 515 .

ORMISTON, G., WOODMAN, D., and LEWIS, F. J. W. (1942), Quart. Med. Jour. ,35, 155 .

\section{PNEUMONIA IN OLD AGE}

\author{
By Trevor H. Howell, M.R.C.P.Ed.
} (Deputy Physician and Surgeon, Royal Hospital,

The statistics of pneumonia in old age are often hard to interpret. In one series collected from past records at the Royal Hospital, Chelsea, the number of deaths from this disease was greater than the number of admissions to the infirmary with the diagnosis. This apparent fallacy is explained by consideration of the clinical course of pneumonia in old people. The majority of cases begin as a mild chronic bronchitis. This has an acute exacerbation, often associated with exposure to cold, fog, or damp. Then complications, such as bronchial spasm or pyrexia, or both, make their appearance. The patient now comes under medical care, is diagnosed as bronchitis and admitted to the infirmary for treatment. A few more obstinate old men try to "shake it off" with proprietary cough mixtures and hot whiskey. But, in a day or two, even these have to report sick. Such patients are often found to have signs of pulmonary consolidation when examined for the first time. They are thus admitted to the infirmary under the diagnosis of broncho-pneumonia. By this time, however, some of the first group of cases have gong on to show pneumonic signs; so that it is not strange for the number of deaths from pneumonias to exceed the number of admissions sometimes.

Among the pneumonias, the lobar type is un usual in the aged, only four cases having been? encountered by the writer in five years. The typical pulmonary consolidation is patchy in the elderly, more often showing signs at the bases andf mid-zones of the lungs than at the apices. Most of the cases show pyrexia, but this is not invariable since a temperature of 98.4 degrees is compatible. with pneumonia in an old person. In some cases this actually represents two degrees of fever, and not normality for such a person. The pulse iso often not much quickened, while the physical signe in the lungs may not always assist the diagnosis in the early stages. Some old men first come toi notice as looking seriously ill, but having a respira $\vec{\sim}$ tion rate of forty as their only demonstrable physical sign. A second group have any signs of pneumonia obscured by widespread rhonchi in ano emphysematous chest. Others only show scatteredP râles all over the lungs, more profuse at the bases than elsewhere for the first day of the disease Both these groups have a raised temperature anct respiration rate in addition to their other signs, as? a warning to the doctor. If left alone, these cases show their consolidation in thirty-six to forty-eighte hours after the rise of temperature. In a series thirty-three Chelsea pensioners with bronctope pneumonia, , sixteen began as bronchitis withs pyrexia. Seven had a sudden onset, with inde응 finite signs in the lungs during the first day of the disease. Eight cases started as apyrexial exacer bations of chronic bronchitis with spasm. The $\overrightarrow{\overrightarrow{0}}$ remaining two men walked about and went to loca $\bar{B}$ public houses for a drink within eight and twelve hours of their deaths, respectively, in spite of extensive pulmonary consolidation when firsto examined.

Bronchial spasm, as shown by rhonchi, is nogु uncommon in cases which are developing pneumonia. Since the treatment of this complication by injection of $\frac{1}{4}-\mathrm{I}$ c.c. of adrenalin, instead of the usual antispasmodic mixture, the outlook of theses patients has improved. The former common sequence, bronchial spasm-pyrexia-broncho․ㅡㄹ. pneumonia is now unusual, even in patients who had shown this pattern once or twice before. The exhibition of sulphapyridine or sulphathiazole tた patients with pyrexial bronchitis, has also appeare to diminish the occurrence of subsequent consolio dation. The opinion of a ward sister at the Royad Hospital, looking back after twenty years, is tha the recovery rate of "full-blown" pneumonias? admitted as such, has not greatly increased. Buto the cases starting as pyrexial or spasmodic bron? 